\title{
Selective Attention Based Graph Convolutional Networks for Aspect-Level Sentiment Classification
}

\author{
Xiaochen Hou*, Jing Huang, Guangtao Wang, Peng Qi, \\ Xiaodong He, Bowen Zhou \\ JD AI Research, Mountain View, CA \\ *xclmm1994@gmail.com
}

\begin{abstract}
Recent work on aspect-level sentiment classification has employed Graph Convolutional Networks (GCN) over dependency trees to learn interactions between aspect terms and opinion words. In some cases, the corresponding opinion words for an aspect term cannot be reached within two hops on dependency trees, which requires more GCN layers to model. However, GCNs often achieve the best performance with two layers, and deeper GCNs do not bring any additional gain. Therefore, we design a novel selective attention based GCN model. On one hand, the proposed model enables the direct interaction between aspect terms and context words via the self-attention operation without the distance limitation on dependency trees. On the other hand, a top- $k$ selection procedure is designed to locate opinion words by selecting $k$ context words with the highest attention scores. We conduct experiments on several commonly used benchmark datasets and the results show that our proposed $S A-G C N$ outperforms strong baseline models.
\end{abstract}

\section{Introduction}

Aspect-level sentiment classification is a finegrained sentiment analysis task, which aims to identify the sentiment polarity (e.g., positive, negative or neutral) of a specific aspect term (also called target) appearing in a sentence. For example, "Despite a slightly limited menu, everything prepared is done to perfection, ultra fresh and a work of food art.", the sentiment polarity of aspect terms "menu" and "food" are negative and positive, respectively. The opinion words "limited" and "done to perfection" provide evidences for sentiment polarity predictions. This task has many applications, such as restaurant recommendation and purchase recommendation on e-commerce websites.

To solve this problem, recent studies have shown that the interactions between an aspect term and its context (which include opinion words) are crucial in identifying the sentiment polarity towards the given term. Most approaches consider the semantic information from the context words and utilize the attention mechanism to learn such interactions. However, it has been shown that syntactic information obtained from dependency parsing is very effective in capturing long-range syntactic relations that are obscure from the surface form (Zhang et al., 2018). A recent popular approach to learn syntaxaware representations is employing graph convolutional networks (GCN) (Kipf and Welling, 2017) model over dependency trees (Huang and Carley, 2019; Zhang et al., 2019; Sun et al., 2019; Wang et al., 2020; Tang et al., 2020), which introduces syntactic inductive biases into the message passing.

In some cases, the most important context words, i.e. opinion words, are more than two-hops away from the aspect term words on the dependency tree. As indicated by Figure 1, there are four hops between the target "Mac OS" and the opinion words "easily picked up" on the dependency tree. This type of cases requires more than two layers of GCN to learn interactions between them. However, previous works show that GCN models with two layers often achieve the best performance (Zhang et al., 2018; Xu et al., 2018), deeper GCNs do not bring additional gain due to the over-smoothing problem (Li et al., 2018b), which makes different nodes have similar representations and lose the distinction among nodes.

In order to solve the above problem, we propose a novel selective attention based GCN (SA-GCN) model, which combines the GCN model over dependency trees with a self-attention based sequence model over the sentence. On one hand, the selfattention sequence model enables the direct interaction between an aspect term and its context so that it can take care of the situation where the term is far away from the opinion words on the dependency tree. On the other hand, a top- $k$ attention selection module is applied after the self-attention opera- 


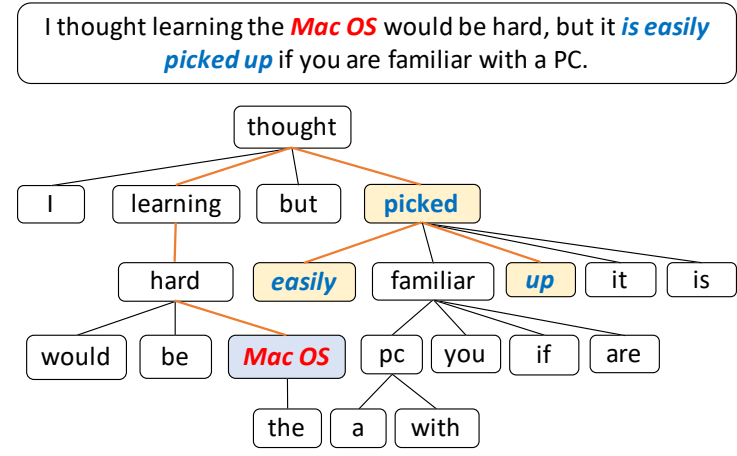

Figure 1: Example of dependency tree with multi-hop between aspect term and determined context words.

tion, which is designed to locate opinion words contained in the context for the aspect term. As shown in Figure 1, if the opinion words "easily picked up" are detected correctly through the top- $k$ selection module, it definitely could help the model to classify the sentiment as positive. To provide supervision information for the top- $k$ selection procedure, we introduce the opinion words extraction task and jointly train the task with the sentiment classification task.

Specifically, the base model is the GCN model over dependency trees. The model uses the pretrained BERT to obtain representations of the aspect term and its context words as the initial node features on the dependency tree.

Next, the GCN outputs are fed into a multi-head top- $k$ attention selection module. For each head, the self-attention operation is applied over the sentence to get a dense attention score matrix, where $i$ th row corresponds the attention scores of all words to the $i$-th word in the sentence. Then for each word, context words with top- $k$ attention scores are selected and others are ignored, which sparsifies the attention score matrix and forms a sparse graph. We design two strategies to get the sparse graph: i) applying top- $k$ selection over the attention matrix obtained by summing attention score matrices of all heads, and thus different heads share the same sparse graph; ii) applying top- $k$ selection on individual attention score matrix of each head, and thus different heads have its own sparse graph. Finally, we apply a GCN layer again to integrate information from such sparse graph(s) for each head, and concatenate the GCN outputs w.r.t. different heads as the final word representation for sentiment analysis.

The main contributions of this work are summarized as the following:

- We propose a selective attention based GCN (SA-
$G C N$ ) module, which takes the benefit of GCN over the dependency trees and enables the aspect term directly obtaining information from the opinion words according to most relevant context words. This helps the model handle cases when the aspect term and opinion words are located far away from each other on the dependency tree.

- We propose to jointly train the sentiment classification and opinion extraction tasks. The joint training further improves the performance of the classification task and provides explanation for sentiment prediction.

\section{Related Work}

Capturing the interaction between the aspect term and opinion words is essential for predicting the sentiment polarity towards the aspect term. In recent work, various attention mechanisms, such as co-attention, self-attention and hierarchical attention, were utilized to learn this interaction (Tang et al., 2016; Liu and Zhang, 2017; Li et al., 2018c; Wang et al., 2018; Fan et al., 2018; Chen et al., 2017; Zheng and Xia, 2018; Wang and Lu, 2018; Li et al., 2018a,c). Specifically, they first encoded the context and the aspect term by recurrent neural networks (RNNs), and then stacked several attention layers to learn the aspect term representations from important context words.

After the success of the pre-trained BERT model (Devlin et al., 2018), Song et al. (2019) utilized the pre-trained BERT as the encoder. In the study by (Xu et al., 2019), the task was considered as a review reading comprehension (RRC) problem. RRC datasets were post trained on BERT and then fine-tuned to the aspect-level sentiment classification. Rietzler et al. (2019) utilized millions of extra data based on BERT to help sentiment analysis.

The above approaches mainly considered the semantic information. Recent approaches attempted to incorporate the syntactic knowledge to learn the syntax-aware representation of the aspect term. Dong et al. (2014) proposed AdaRNN, which adaptively propagated the sentiments of words to target along the dependency tree in a bottom-up manner. Nguyen and Shirai (2015) extended RNN to obtain the representation of the target aspect by aggregating the syntactic information from the dependency and constituent tree of the sentence. $\mathrm{He}$ et al. (2018) proposed to use the distance between the context word and the aspect term along the dependency tree as the attention weight. Some re- 
searchers (Huang and Carley, 2019; Zhang et al., 2019; Sun et al., 2019) employed GNNs over dependency trees to aggregate information from syntactic neighbors. Most recent work in Wang et al. (2020) proposed to reconstruct the dependency tree to an aspect-oriented tree. The reshaped tree only kept the dependency structure around the aspect term and got rid of all other dependency connections, which made the learned node representations not fully syntax-aware. Tang et al. (2020) designed a mutual biaffine module between Transformer encoder and the GCN encoder to enhance the representation learning.

The downside of applying GCN over dependency trees is that it cannot elegantly handle the long distance between aspect terms and opinion words. Our proposed $S A-G C N$ model effectively integrates the benefit of a GCN model over dependency trees and a self-attention sequence model to directly aggregate information from opinion words The top- $k$ self-attention sequence model selects the most important context words, which effectively sparsifies the fully-connected graph from self-attention. Then we apply another GCN layer on top of this new sparsified graph, such that each of those important context words is directly reachable by the aspect term and the interaction between them could be learned.

\section{Proposed Model}

\subsection{Overview of the Model}

The goal of our proposed $S A-G C N$ model is to predict the sentiment polarity of an aspect term in a given sentence. To improve the sentiment classification performance and provide explanations about the polarity prediction, we also introduce the opinion extraction task for joint training. The opinion extraction task aims to predict a tag sequence $\boldsymbol{y}_{o}=\left[y_{1}, y_{2}, \cdots, y_{n}\right]\left(y_{i} \in\{B, I, O\}\right)$ denotes the beginning of, inside of, and outside of opinion words. Figure 2 illustrates the overall architecture of the $S A-G C N$ model. For each instance composing of a sentence-term pair, all the words in the sentence except for the aspect term are defined as context words.

\subsection{Encoder for Aspect Term and Context}

BERT Encoder. We use the pre-trained BERT base model as the encoder to obtain embeddings of sentence words. Suppose a sentence consists of $n$ words $\left\{w_{1}, w_{2}, \ldots, w_{\tau}, w_{\tau+1} \ldots, w_{\tau+m}, \ldots, w_{n}\right\}$ where $\left\{w_{\tau}, w_{\tau+1} \ldots, w_{\tau+m-1}\right\}$ stand for the aspect term containing $m$ words. First, we construct the input as "[CLS] + sentence + [SEP] + term + [SEP]" and feed it into BERT. This input format enables explicit interactions between the whole sentence and the term such that the obtained word representations are term-attended. Then, we use average pooling to summarize the information carried by sub-words from BERT and obtain final embeddings of words $\boldsymbol{X} \in \mathbb{R}^{n \times d_{B}}, d_{B}$ refers to the dimensionality of BERT output.

\subsection{GCN over Dependency Trees}

With words representations $\boldsymbol{X}$ as node features and dependency tree as the graph, we employ a GCN to capture syntactic relations between the term node and its neighboring nodes.

GCNs have shown to be effective for many NLP applications, such as relation extraction (Guo et al., 2019; Zhang et al., 2018), reading comprehension (Kundu et al., 2019; Tu et al., 2019), and aspect-level sentiment analysis (Huang and Carley, 2019; Zhang et al., 2019; Sun et al., 2019). In each GCN layer, a node aggregates the information from its one-hop neighbors and update its representation. In our case, the graph is represented by the dependency tree, where each word is treated as a single node and its representation is denoted as the node feature. The message passing on the graph can be formulated as follows:

$$
\boldsymbol{H}^{(l)}=\sigma\left(\boldsymbol{A} \boldsymbol{H}^{(l-1)} \boldsymbol{W}^{(l-1)}\right)
$$

where $\boldsymbol{H}^{(l)} \in \mathbb{R}^{n \times d_{h}}$ is the output $l$-th GCN layer, $\boldsymbol{H}^{(0)} \in \mathbb{R}^{n \times d_{B}}$ is the input of the first GCN layer, and $\boldsymbol{H}^{(0)}=\boldsymbol{X} \in \mathbb{R}^{n \times d_{B}} . \boldsymbol{A} \in \mathbb{R}^{n \times n}$ denotes the adjacency matrix obtained from the dependency tree, note that we add a self-loop on each node. $\boldsymbol{W}$ represents the learnable weights, where $\boldsymbol{W}^{(0)} \in$ $\mathbb{R}^{d_{B} \times d_{h}}$ and $\boldsymbol{W}^{(l-1)} \in \mathbb{R}^{d_{h} \times d_{h}} . \sigma$ refers to $R e L U$ activation function.

The node features are passed through the GCN layer, the representation of each node is now further enriched by syntax information from the dependency tree.

\subsection{SA-GCN: Selective Attention based GCN}

Although performing GCNs over dependency trees brings syntax information to the representation of each word, it could still limit interactions between aspect terms and long-distance opinion words that are essential for determining the sentiment polarity. In order to alleviate the problem, we apply a 


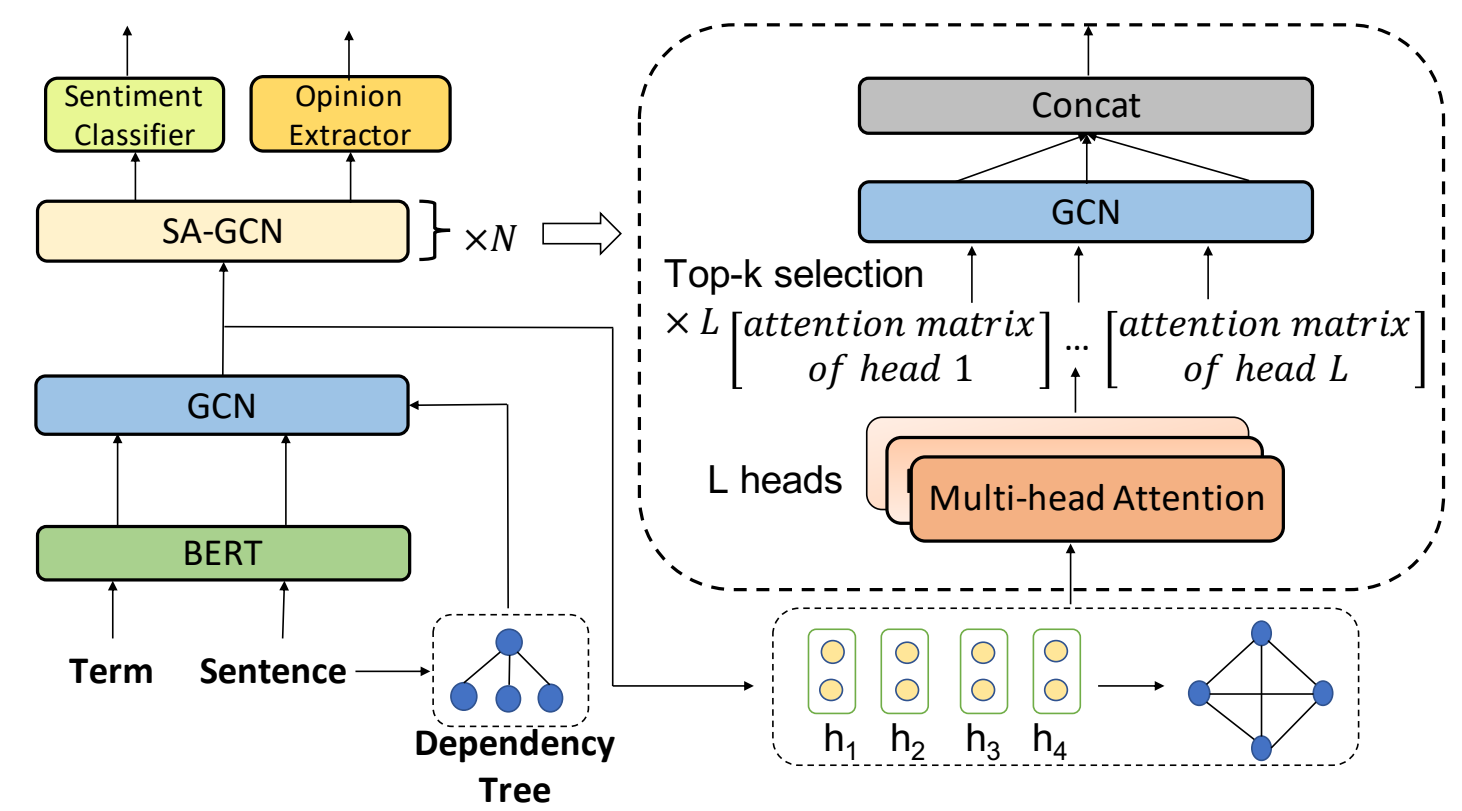

Figure 2: The $S A-G C N$ model architecture: the left part is the overview of the framework, the right part shows details of a $S A-G C N$ block.

Selective Attention based GCN $(S A-G C N)$ block to identify the most important context words and integrate their information into the representation of the aspect term. Multiple $S A-G C N$ blocks can be stacked to form a deep model. Each $S A-G C N$ block is composed of three parts: a multi-head selfattention layer, top- $k$ selection and a GCN layer.

Self-Attention. We apply the multi-head selfattention first to get the attention score matrices $\boldsymbol{A}_{\text {score }}^{i} \in \mathbb{R}^{n \times n}(1 \leq i \leq L), L$ is the number of heads. It can be formulated as:

$$
\begin{aligned}
\boldsymbol{A}_{\text {score }}^{i} & =\frac{\left(\boldsymbol{H}_{k, i} \boldsymbol{W}_{k}\right)\left(\boldsymbol{H}_{q, i} \boldsymbol{W}_{q}\right)^{T}}{\sqrt{d_{\text {head }}}} \\
d_{\text {head }} & =\frac{d_{h}}{L}
\end{aligned}
$$

where $\boldsymbol{H}_{*, i}=\boldsymbol{H}_{*}[:,:, i], * \in\{k:$ key, $q$ : query $\}$, $\boldsymbol{H}_{k} \in \mathbb{R}^{n \times d_{\text {head }} \times L}$ and $\boldsymbol{H}_{q} \in \mathbb{R}^{n \times d_{\text {head }} \times L}$ are the node representations from the previous GCN layer, $\boldsymbol{W}_{k} \in \mathbb{R}^{d_{\text {head }} \times d_{\text {head }}}$ and $\boldsymbol{W}_{q} \in \mathbb{R}^{d_{\text {head }} \times d_{\text {head }} \text { are }}$ learnable weight matrices, $d_{h}$ is the dimension of the input node feature, and $d_{\text {head }}$ is the dimension of each head.

The obtained attention score matrices can be considered as $L$ fully-connected (complete) graphs, where each word is connected to all the other context words with different attention weights. This kind of attention score matrix has been used in attention-guided GCNs for relation extraction (Guo et al., 2019). Although the attention weight is help- ful to differentiate different words, the fully connected graph still results in the aspect node fusing all the other words information directly, and the noise is often introduced during feature aggregation in GCNs, which further hurts the sentiment prediction. Therefore, we propose a top- $k$ attention selection mechanism to sparsify the fully connected graph, and obtain a new sparse graph for feature aggregation for GCN. This is different from attentionguided GCNs (Guo et al., 2019) which performed feature aggregation over the fully-connected graph. Moreover, our experimental study (see Table 5 in Section 4) also confirms that the top- $k$ selection is quite important and definitely beneficial to the aspect-term classification task.

Top- $k$ Selection. For each attention score matrix $\boldsymbol{A}_{\text {score }}^{i}$, we find the top- $k$ important context words for each word, which effectively remove some edges in $\boldsymbol{A}_{\text {score }}^{i}$. The reason why we choose the top- $k$ context words is that only a few words are sufficient to determine the sentiment polarity towards an aspect term. Therefore, we discard other words with low attention scores to get rid of irrelevant noisy words.

We design two strategies for top- $k$ selection, head-independent and head-dependent. Headindependent selection determines $k$ context words by aggregating the decisions made by all heads and reaches to an agreement among heads, while headdependent policy enables each head to keep its own 
selected $k$ words.

Head-independent selection is defined as following: we first sum the attention score matrix of each head element-wise, and then find top- $k$ context words using the mask generated by the function topk. For example, topk $([0.3,0.2,0.5])$ returns $[1,0,1]$ if $k$ is set to 2 . Finally, we apply a softmax operation on the updated attention score matrix. The process could be formulated as follows:

$$
\begin{aligned}
\boldsymbol{A}_{\text {sum }} & =\sum_{i=1}^{L} \boldsymbol{A}_{\text {score }}^{i} \\
\boldsymbol{A}_{m_{\text {ind }}} & =\operatorname{topk}\left(\boldsymbol{A}_{\text {sum }}\right) \\
\boldsymbol{A}_{h_{\text {ind }}}^{i} & =\operatorname{softmax}\left(\boldsymbol{A}_{m_{\text {ind }}} \circ \boldsymbol{A}_{\text {score }}^{i}\right)
\end{aligned}
$$

where $\boldsymbol{A}_{\text {score }}^{i}$ is the attention score matrix of $i$-th head, o denotes the element-wise multiplication.

Head-dependent selection finds top- $k$ context words according to the attention score matrix of each head individually. We apply the softmax operation on each top- $k$ attention matrix. This step can be formulated as:

$$
\begin{aligned}
\boldsymbol{A}_{m_{\text {dep }}}^{i} & =\operatorname{topk}\left(\boldsymbol{A}_{\text {score }}^{i}\right) \\
\boldsymbol{A}_{h_{\text {dep }}}^{i} & =\operatorname{softmax}\left(\boldsymbol{A}_{m_{\text {dep }}}^{i} \circ \boldsymbol{A}_{\text {score }}^{i}\right)
\end{aligned}
$$

Compared to head-independent selection with exactly $k$ words selected, head-dependent usually selects a larger number (than $k$ ) of important context words. Because each head might choose different $k$ words thus more than $k$ words are selected in total.

From top- $k$ selection we obtain $L$ graphs based on the new attention scores and pass them to the next GCN layer. For simplicity, we will omit the head-ind and head-dep subscript in the later section. The obtained top- $k$ score matrix $A$ could be treated as an adjacency matrix, where $\boldsymbol{A}(p, q)$ denotes as the weight of the edge connecting word $p$ and word $q$. Note that $\boldsymbol{A}$ does not contain self-loop, and we add a self-loop for each node.

GCN Layer. After top- $k$ selection on each attention score matrix $\boldsymbol{A}_{\text {score }}^{i}$ ( $\boldsymbol{A}_{\text {score }}^{i}$ is not fully connected anymore), we apply a one-layer GCN and get updated node features as follows:

$$
\begin{aligned}
\hat{\boldsymbol{H}}^{(l, i)} & =\sigma\left(\boldsymbol{A}^{i} \hat{\boldsymbol{H}}^{(l-1)} \boldsymbol{W}^{i}\right)+\hat{\boldsymbol{H}}^{(l-1)} \boldsymbol{W}^{i} \\
\hat{\boldsymbol{H}}^{(l)} & =\|_{i=1}^{L} \hat{\boldsymbol{H}}^{(l, i)}
\end{aligned}
$$

where $\hat{\boldsymbol{H}}^{(l)} \in \mathbb{R}^{n \times d_{h}}$ is the output of the $l$-th $S A$ $G C N$ block and composed by the concatenation of $\hat{\boldsymbol{H}}^{(l, i)} \in \mathbb{R}^{n \times d_{\text {head }}}$ of $i$-th head, $\hat{\boldsymbol{H}}^{(0)} \in \mathbb{R}^{n \times d_{h}}$ is the input of the first $S A-G C N$ block and comes from the GCN layer operating on the dependency tree, $\boldsymbol{A}^{i}$ is the top- $k$ score matrix of $i$-th head, $\boldsymbol{W}^{i} \in$

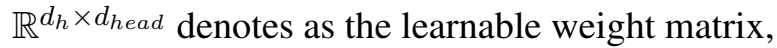
and $\sigma$ refers to $R e L U$ activation function. The $S A$ $G C N$ block can be applied multi times if needed.

\subsection{Classifier}

Based on the output $\hat{\boldsymbol{H}}_{O}$ of the last $S A-G C N$ block, we extract the aspect term node features from $\hat{\boldsymbol{H}}_{o}$, and conduct average pooling to obtain the aspect term ${ }^{1}$ representation $\hat{\boldsymbol{h}}_{t} \in \mathbb{R}^{1 \times d_{h}}$. Then we feed it into a two-layer MLP to calculate the final classification scores $\hat{\boldsymbol{y}}_{s}$ :

$$
\hat{\boldsymbol{y}}_{s}=\operatorname{softmax}\left(\boldsymbol{W}_{2} \sigma\left(\boldsymbol{W}_{1} \hat{\boldsymbol{h}}_{t}^{T}\right)\right)
$$

where $\boldsymbol{W}_{2} \in \mathbb{R}^{C \times d_{\text {out }}}$ and $\boldsymbol{W}_{1} \in \mathbb{R}^{d_{\text {out }} \times d_{h}}$ denote the learnable weight matrix, $C$ is the sentiment class number, and $\sigma$ refers to $R e L U$ activation function. We use cross entropy as the sentiment classification loss function:

$$
L_{s}=-\sum_{c=1}^{C} \boldsymbol{y}_{s, c} \log \hat{\boldsymbol{y}}_{s, c}+\lambda\|\theta\|^{2}
$$

where $\lambda$ is the coefficient for L2-regularization, $\theta$ denotes the parameters that need to be regularized, $\boldsymbol{y}_{s}$ is the true sentiment label.

\subsection{Opinion Extractor}

The opinion extraction shares the same input encoder, i.e. the $S A-G C N$ as sentiment classification. Therefore we feed the output of $S A-G C N$ to a linearchain Conditional Random Field (CRF) (Lafferty et al., 2001), which is the opinion extractor. Specifically, based on the $S A-G C N$ output $\hat{\boldsymbol{H}}_{o}$, the output sequence $\boldsymbol{y}_{o}=\left[y_{1}, y_{2}, \cdots, y_{n}\right]\left(y_{i} \in\{B, I, O\}\right)$ is predicted as:

$$
\begin{aligned}
p\left(\boldsymbol{y}_{o} \mid \hat{\boldsymbol{H}}_{o}\right) & =\frac{\exp \left(s\left(\hat{\boldsymbol{H}}_{o}, \boldsymbol{y}_{o}\right)\right)}{\sum_{\boldsymbol{y}_{o}{ }_{o} \in Y} \exp \left(s\left(\hat{\boldsymbol{H}}_{o}, \boldsymbol{y}_{o}^{\prime}\right)\right)} \\
s\left(\hat{\boldsymbol{H}}_{o}, \boldsymbol{y}_{o}\right) & =\sum_{i}^{n}\left(\boldsymbol{T}_{y_{i-1}, y_{i}}+\boldsymbol{P}_{i, y_{i}}\right) \\
\boldsymbol{P}_{i} & =\boldsymbol{W}_{o} \hat{\boldsymbol{H}}_{o}[i]+\boldsymbol{b}_{o}
\end{aligned}
$$

where $Y$ denotes the set of all possible tag sequences, $\boldsymbol{T}_{y_{i-1}, y_{i}}$ is the transition score matrix, $\boldsymbol{W}_{o}$ and $\boldsymbol{b}_{o}$ are learnable parameters. We apply Viterbi

\footnotetext{
${ }^{1}$ The aspect term might be composed of multiple term nodes in the graph.
} 


\begin{tabular}{ccccccc}
\hline \multirow{2}{*}{ Dataset } & \multicolumn{2}{c}{ Positive } & \multicolumn{2}{c}{ Neutral } & \multicolumn{2}{c}{ Negative } \\
\cline { 2 - 7 } & Train & Test & Train & Test & Train & Test \\
\hline 14Lap & 991 & 341 & 462 & 169 & 867 & 128 \\
14Rest & 2164 & 728 & 633 & 196 & 805 & 196 \\
15Rest & 963 & 353 & 36 & 37 & 280 & 207 \\
16Rest & 1324 & 483 & 71 & 32 & 489 & 135 \\
\hline
\end{tabular}

Table 1: Statistics of Datasets.

algorithm in the decoding phase. And the loss for opinion extraction task is defined as:

$$
L_{o}=-\log \left(p\left(\boldsymbol{y}_{o} \mid \hat{\boldsymbol{H}}_{o}\right)\right)
$$

Finally, the total training loss is:

$$
L=L_{s}+\alpha L_{o}
$$

where $\alpha \geq 0$ represents the weight of opinion extraction task.

\section{Experiments}

Data Sets. We evaluate our $S A-G C N$ model on four datasets: Laptop reviews from SemEval 2014 Task 4 (14Lap), Restaurant reviews from SemEval 2014 Task 4 (Pontiki et al., 2014), SemEval 2015 Task 12 (Pontiki et al., 2015) and SemEval 2016 Task 5 (Pontiki et al., 2016) (14Rest, 15Rest and 16Rest). We remove several examples with "conflict" labels. The statistics of these datasets are listed in Table 1. The opinion words labeling for these four datasets come from (Fan et al., 2019).

Baselines. Since BERT(Devlin et al., 2018) model shows significant improvements over many NLP tasks, we directly implement $S A-G C N$ based on BERT and compare with following BERT-based baseline models:

1. BERT-SPC (Song et al., 2019) feeds the sentence and term pair into the BERT model and the BERT outputs are used for prediction.

2. AEN-BERT (Song et al., 2019) uses BERT as the encoder and employs several attention layers.

3. TD-GAT-BERT (Huang and Carley, 2019) utilizes GAT on the dependency tree to propagate features from the syntactic context.

4. DGEDT-BERT (Tang et al., 2020) proposes a mutual biaffine module to jointly consider the flat representations learnt from Transformer and graph-based representations learnt from the corresponding dependency graph in an iterative manner.
5. R-GAT+BERT (Wang et al., 2020) reshapes and prunes the dependency parsing tree to an aspectoriented tree rooted at the aspect term, and then employs relational GAT to encode the new tree for sentiment predictions.

In our experiments, we present results of the average and standard deviation numbers from seven runs of different random initialization. We use BERT-base model to compare with other published numbers. We implement our own BERT-baseline by directly applying a classifier on top of BERTbase encoder, BERT+2-layer GCN and BERT+4layer $G C N$ are models with 2-layer and 4-layer GCN respectively on dependency trees with the BERT encoder. BERT+SA-GCN is our proposed $S A-G C N$ model with BERT encoder. Joint $S A-G C N$ refers to joint training of sentiment classification and opinion extraction tasks.

Evaluation metrics. We train the model on training set, and evaluate the performance on test set in terms of accuracy and macro-F1 scores which are commonly-used in sentiment analysis (Sun et al., 2019; Tang et al., 2016; Wang et al., 2020).

Parameter Setting. During training, we set the learning rate to $10^{-5}$. The batch size is 4 . We train the model up to 5 epochs with Adam optimizer. We obtain dependency trees using the Stanford Stanza (Qi et al., 2020). The dimension of BERT output $d_{B}$ is 768 . The hidden dimensions are selected from $\{128,256,512\}$. We apply dropout (Srivastava et al., 2014) and the dropout rate range is $[0.1,0.4]$. The $\mathrm{L} 2$ regularization is set to $10^{-6}$. We use 1 or $2 S A-G C N$ blocks in our experiments. We choose $k$ in top- $k$ selection module from $\{2,3\}$ to achieve the best performance. For joint training, the weight range of opinion extraction loss is $[0.05,0.15]^{2}$

\subsection{Experimental Results}

We present results of the $S A-G C N$ model in two aspects: classification performance and qualitative case study.

Classification. Table 2 shows comparisons of $S A$ $G C N$ with other baselines in terms of classification accuracy and Macro-F1. From this table, we observe that: $S A-G C N$ achieves the best average results on 14Lap, 15Rest and 16Rest datasets, and obtains competitive results on 14Rest dataset. The joint training of sentiment classification and opin-

\footnotetext{
${ }^{2}$ Our code will be released at the time of publication.
} 


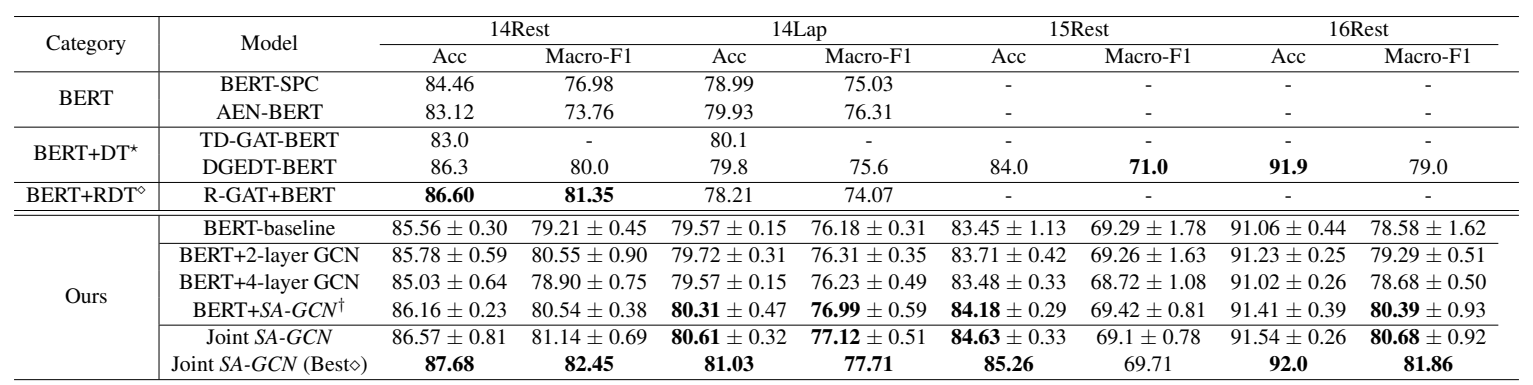

$\star$ DT: Dependency Tree; $\diamond$ RDT: Reshaped Dependency Tree.

$\dagger$ : Head-independent based top- $k$ Selection.

$\diamond$ The "best" denotes as the best performances of our SA-GCN model from the seven runs. Row "Joint-SA-GCN" reports the average and std of these seven runs.

Table 2: Comparison of $S A-G C N$ with various baselines.

\begin{tabular}{l|l}
\hline Sentence & Label \\
\hline Satay is one of those favorite haunts on Washington where the service and food is always on the money. & positive neutral positive \\
\hline And the fact that it comes with an i5 processor definitely speeds things up & positive neutral positive \\
\hline I know real Indian food and this was n't it. & negative neutral negative \\
\hline
\end{tabular}

Table 3: Top- $k$ visualization: the darker the shade, the larger attention weight.

ion extraction tasks further boosts the performances on all datasets.

Specifically, BERT+2-layer $G C N$ outperforms $B E R T$-baseline, which proves the benefit of using syntax information. BERT+4-layer $G C N$ is actually worse than $B E R T+2$-layer $G C N$, which shows that more GCN layers do not bring additional gain.

Our BERT+SA-GCN model further outperforms the BERT+2-layer GCN model. Because the $S A$ $G C N$ block allows aspect terms to directly absorb the information from the most important context words that are not reachable within two hops in the dependency tree.

Besides, introducing the opinion extraction task provides more supervision signals for the top- $k$ selection module, which benefits the sentiment classification task.

Qualitative Case Study. To show the efficacy of the $S A-G C N$ model on dealing long-hops between aspect term and its opinion words, we demonstrate three examples as shown in Table 3. These sentences are selected from test sets of 14Lap and 14Rest datasets and predicted correctly by the $S A$ $G C N$ model but wrongly by BERT+2-layer $G C N$. The important thing to note here, our $S A-G C N$ model could provide explanation about the prediction according to the learned attention weights, while the GCN based model (BERT+2-layer GCN denoted as "GCN" in Table 3) cannot. Aspect terms are colored red. Top-3 words with the largest attention weights towards the aspect term are shaded. The darker the shade, the larger attention weight.

In all three examples the aspect terms are more than three hops away from essential opinion words

\begin{tabular}{l|cccc}
\hline \multirow{2}{*}{ Model } & 14Rest & 14Lap & 15Rest & 16Rest \\
\cline { 2 - 5 } & F1 & F1 & F1 & F1 \\
\hline IOG & 80.24 & 71.39 & 73.51 & 81.84 \\
ASTE & 83.15 & 76.03 & 78.02 & 83.73 \\
\hline Joint $S A-G C N$ & $\mathbf{8 3 . 7 2} \pm 0.51$ & $\mathbf{7 6 . 7 9} \pm 0.33$ & $\mathbf{8 0 . 9 9} \pm 0.43$ & $\mathbf{8 3 . 8 3} \pm 0.50$ \\
\hline
\end{tabular}

Table 4: Opinion extraction results.

(Please refer to Fig. 3), thus BERT+2-layer GCN model cannot learn the interactions between them within two layers, while $S A-G C N$ model overcomes the distance limitation and locates right opinion words.

Opinion Extraction. Table 4 shows the results of the opinion extraction task under the joint training setting. The reported numbers are obtained by averaging $\mathrm{F} 1$ of seven runs. In each run, the selected opinion $\mathrm{F} 1$ is generated from the best sentiment classification checkpoint. We compare our model with two baselines: IOG (Fan et al., 2019) encodes the aspect term information into context by an Inward-Outward LSTM to find the corresponding opinion words. ASTE (Peng et al., 2020) utilizes a GCN module to learn the mutual dependency relations between different words and to guide opinion term extraction. As shown in this table, the joint $S A$ $G C N$ model outperforms two baseline models on all datasets, which demonstrates that the sentiment classification task is helpful for opinion extraction task as well.

\subsection{Model Analysis}

We further analyze our $S A-G C N$ model from two perspectives: ablation study and sentence length analysis.

Ablation Study. To demonstrate effectiveness of different modules in $S A-G C N$, we conduct ablation 


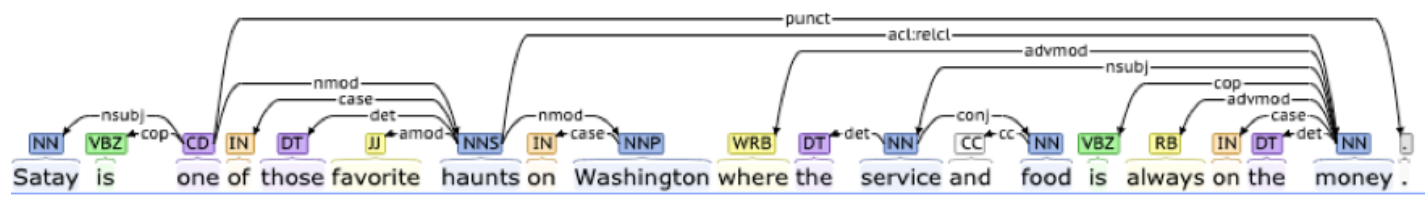

(a) case 1

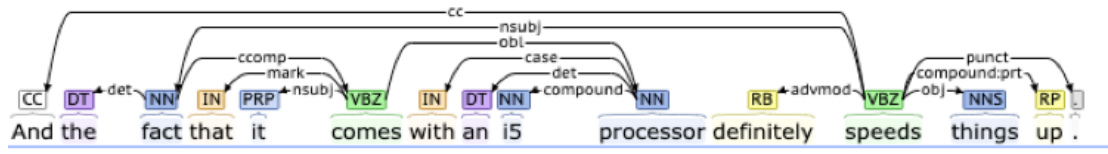

(b) case 2

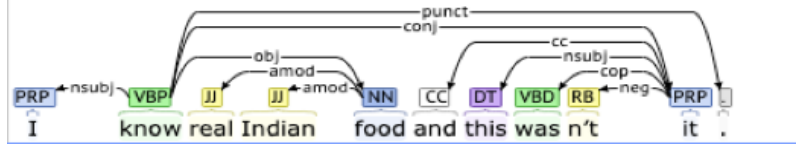

(c) case 3

Figure 3: Dependency trees of case study. Case 1: the aspect term "food" is four hops away from the opinion words "favorite" and "on the money". In cases 2 and 3, there are also three-hops distance between aspect terms and opinion words.

\begin{tabular}{|c|c|c|c|c|c|c|c|c|}
\hline \multirow{2}{*}{ Model } & \multicolumn{2}{|c|}{ 14Rest } & \multicolumn{2}{|c|}{ 14Lap } & \multicolumn{2}{|c|}{ 15Rest } & \multicolumn{2}{|c|}{ 16Rest } \\
\hline & Acc & Macro-F1 & Acc & Macro-F1 & Acc & Macro-F1 & Acc & Macro-F1 \\
\hline$S A-G C N$ (head-ind) & $86.16 \pm 0.23$ & $\mathbf{8 0 . 5 4} \pm 0.38$ & $\mathbf{8 0 . 3 1} \pm 0.47$ & $76.99 \pm 0.59$ & $84.18 \pm 0.29$ & $69.42 \pm 0.81$ & $91.41 \pm 0.39$ & $\overline{80.39} \pm 0.93$ \\
\hline$S A-G C N$ w/o top-k & $85.06 \pm 0.68$ & $78.88 \pm 0.83$ & $79.96 \pm 0.14$ & $76.64 \pm 0.58$ & $83.15 \pm 0.41$ & $68.74 \pm 1.48$ & $90.92 \pm 0.45$ & $78.18 \pm 0.71$ \\
\hline$S A-G C N\left(\right.$ head-dep) ${ }^{\diamond}$ & $85.41 \pm 0.21$ & $79.19 \pm 0.68$ & $80.17 \pm 0.55$ & $76.83 \pm 0.59$ & $83.68 \pm 0.54$ & $68.81 \pm 1.39$ & $91.01 \pm 0.40$ & $78.88 \pm 1.04$ \\
\hline
\end{tabular}

Table 5: Ablation study of $S A-G C N$.

studies in Table 5. From this table, we observe that:

1. Effect of Top-k Selection. To examine the impact the top-k selection, we present the result of $S A-G C N$ w/o top- $k$ in Table 5. We can see that without top-k selection, both accuracy and macro-F1 decrease on all datasets. This observation proves that the top-k selection helps to reduce the noisy context and locate top important opinion words. We also conduct the effect of the hyper-parameter $k$ and the block number $N$ on $S A-G C N$ under head-independent and head-dependent selection respectively (see the supplemental material).

2. Effect of Head-independent and Headdependent Selection. As shown in the last row in Table 5, head-independent selection achieves better results than head-dependent selection. This is because the mechanism of head-independent selection is similar to voting. By summing up the weight scores from each head, context words with higher scores in most heads get emphasized, and words that only show importance in few heads are filtered out. Thus all heads reach to an agreement and the top- $k$ context words are decided. However for head- dependent selection, each head selects different top- $k$ context words, which is more likely to choose certain unimportant context words and introduce noise to the model prediction.

Sentence Length Analysis. To quantify the ability of our $S A-G C N$ model dealing with long-distance problem, we conduct sentence length analysis on 14Lap and 14Rest datasets. The assumption is that the longer the sentence, the more likely the longdistance problem occurs. The results are showed in Figure 4. We measure the sentiment classification accuracy of BERT+2-layer GCN (denotes as GCN in Figure 4) and BERT+SA-GCN models under different sentence lengths. We observe that $S A$ $G C N$ achieves better accuracy than GCN across all length ranges and is more advantageous when sentences are longer. To some extent, the results prove effectiveness of $S A-G C N$ in dealing with long-distance problem.

Hyper-parameter Analysis. We examine the effect of the hype-parameter $k$ and the block number $N$ on our proposed model under head-independent and head-dependent selection respectively. Figure 5 shows the results on 14Rest dataset.

1. Effect of Hyper-parameter $k$. From Figure 


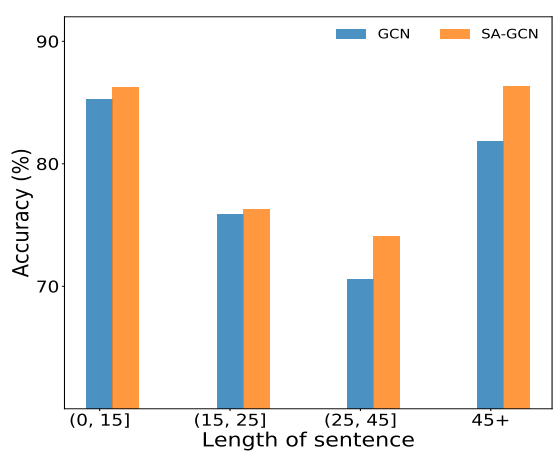

(a) Length analysis on 14Lap.

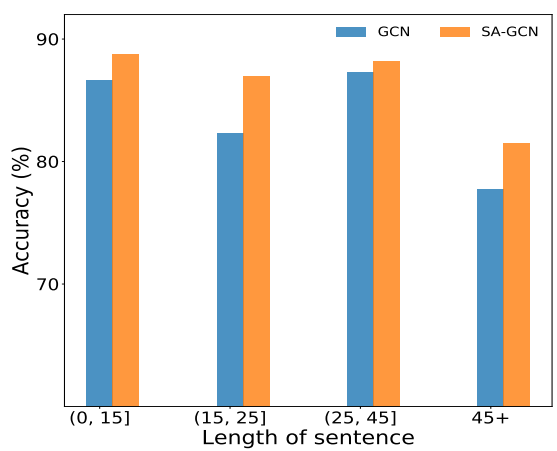

(b) Length analysis on 14Rest.

Figure 4: Sentence length analysis on 14Lap and 14Rest.

5a, we observe that: 1) the highest accuracy appears when $k$ is equal to 3 . As $k$ becomes bigger, the accuracy goes down. The reason is that integrating information from too many context words could introduce distractions and confuse the representation of the current word. 2) Head-independent selection performs better than head-dependent selection as $k$ increases. As mentioned before, compared with head-independent, head-dependent selection might have more than $k$ context words contribute to the aggregation and introduce some noise.

2. Effect of Block Number. Figure $5 b$ shows the effect of different number of $S A-G C N$ blocks. As the block number increases, the accuracy decreases for both head-independent and headdependent selection. A single $S A-G C N$ block is sufficient for selecting top- $k$ important context words. Stacking multiple blocks introduces more parameters and thus would lead to overfitting with such a small amount of training data. This might be the reason why stacking multiple blocks is not helpful. For our future work we plan to look into suitable deeper GNN models that are good for this task.

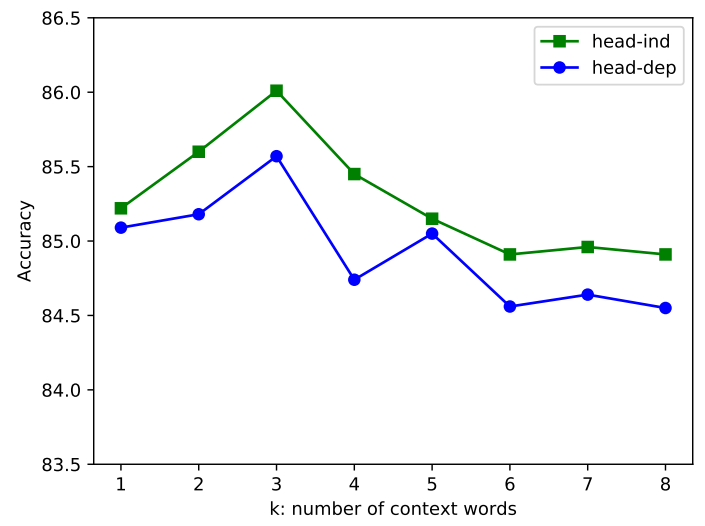

(a) Impact of $k$

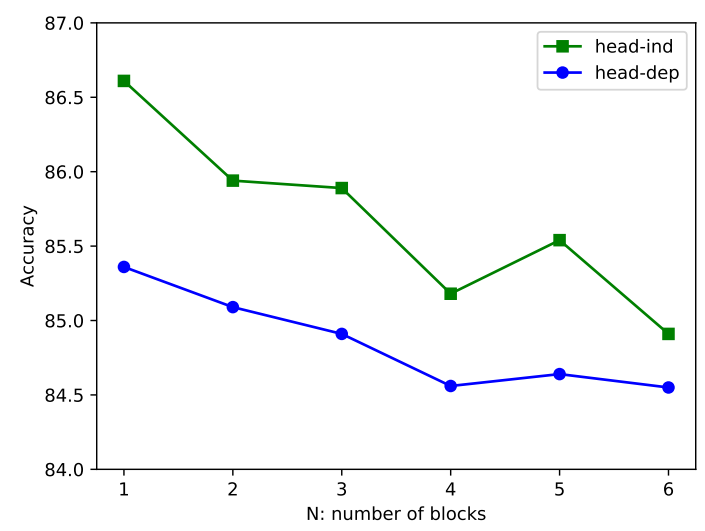

(b) Impact of block numbers

Figure 5: Impact of $k$ and block numbers on $S A-G C N$ over Restaurant dataset.

\section{Conclusions}

We propose a selective attention based GCN model for the aspect-level sentiment classification task. We first encode the aspect term and context words by pre-trained BERT to capture the interaction between them, then build a GCN on the dependency tree to incorporate syntax information. In order to handle the long distance between aspect terms and opinion words, we use the selective attention based GCN block, to select the top- $k$ important context words and employ the GCN to integrate their information for the aspect term representation learning. Further, we adopt opinion extraction problem as an auxiliary task to jointly train with sentiment classification task. We conduct experiments on several SemEval datasets. The results show that $S A-G C N$ achieve better performances than previous strong baselines.

\section{References}

Peng Chen, Zhongqian Sun, Lidong Bing, and Wei Yang. 2017. Recurrent attention network on memory for aspect sentiment analysis. In Proceedings of 
the 2017 conference on empirical methods in natural language processing, pages 452-461.

Jacob Devlin, Ming-Wei Chang, Kenton Lee, and Kristina Toutanova. 2018. Bert: Pre-training of deep bidirectional transformers for language understanding. Proceedings of NAACL-HLT 2019, page pages 4171-4186.

Li Dong, Furu Wei, Chuanqi Tan, Duyu Tang, Ming $\mathrm{Zhou}$, and $\mathrm{Ke} \mathrm{Xu}$. 2014. Adaptive recursive neural network for target-dependent twitter sentiment classification. In Proceedings of the 52nd annual meeting of the association for computational linguistics (volume 2: Short papers), pages 49-54.

Feifan Fan, Yansong Feng, and Dongyan Zhao. 2018. Multi-grained attention network for aspect-level sentiment classification. In Proceedings of the 2018 Conference on Empirical Methods in Natural Language Processing, pages 3433-3442.

Zhifang Fan, Zhen Wu, Xinyu Dai, Shujian Huang, and Jiajun Chen. 2019. Target-oriented opinion words extraction with target-fused neural sequence labeling. In Proceedings of the 2019 Conference of the North American Chapter of the Association for Computational Linguistics: Human Language Technologies, Volume 1 (Long and Short Papers), pages 2509-2518.

Zhijiang Guo, Yan Zhang, and Wei Lu. 2019. Attention guided graph convolutional networks for relation extraction. 57th Annual Meeting of the Association for Computational Linguistics, page 241-251.

Ruidan He, Wee Sun Lee, Hwee Tou Ng, and Daniel Dahlmeier. 2018. Effective attention modeling for aspect-level sentiment classification. In Proceedings of the 27th International Conference on Computational Linguistics, pages 1121-1131.

Binxuan Huang and Kathleen M Carley. 2019. Syntaxaware aspect level sentiment classification with graph attention networks. In Proceedings of the 2019 Conference on Empirical Methods in Natural Language Processing and the 9th International Joint Conference on Natural Language Processing (EMNLP-IJCNLP), pages 5472-5480.

Thomas N. Kipf and Max Welling. 2017. Semisupervised classification with graph convolutional networks. In International Conference on Learning Representations (ICLR).

Souvik Kundu, Tushar Khot, Ashish Sabharwal, and Peter Clark. 2019. Exploiting explicit paths for multi-hop reading comprehension. In Proceedings of the 57th Annual Meeting of the Association for Computational Linguistics, pages 2737-2747. Association for Computational Linguistics.

John Lafferty, Andrew McCallum, and Fernando CN Pereira. 2001. Conditional random fields: Probabilistic models for segmenting and labeling sequence data.
Lishuang Li, Yang Liu, and AnQiao Zhou. 2018a. Hierarchical attention based position-aware network for aspect-level sentiment analysis. In Proceedings of the 22nd Conference on Computational Natural Language Learning, pages 181-189.

Qimai Li, Zhichao Han, and Xiao-Ming Wu. 2018b. Deeper insights into graph convolutional networks for semi-supervised learning. In Thirty-Second AAAI Conference on Artificial Intelligence.

Xin Li, Lidong Bing, Wai Lam, and Bei Shi. 2018c. Transformation networks for target-oriented sentiment classification. In Proceedings of the 56th Annual Meeting of the Association for Computational Linguistics (Volume 1: Long Papers), pages 946956, Melbourne, Australia. Association for Computational Linguistics.

Jiangming Liu and Yue Zhang. 2017. Attention modeling for targeted sentiment. In Proceedings of the 15th Conference of the European Chapter of the Association for Computational Linguistics: Volume 2, Short Papers, pages 572-577.

Thien Hai Nguyen and Kiyoaki Shirai. 2015. Phrasernn: Phrase recursive neural network for aspect-based sentiment analysis. In Proceedings of the 2015 Conference on Empirical Methods in Natural Language Processing, pages 2509-2514.

Haiyun Peng, Lu Xu, Lidong Bing, Fei Huang, Wei Lu, and Luo Si. 2020. Knowing what, how and why: A near complete solution for aspect-based sentiment analysis. In $A A A I$, pages $8600-8607$.

Maria Pontiki, Dimitris Galanis, Haris Papageorgiou, Ion Androutsopoulos, Suresh Manandhar, Mohammad AL-Smadi, Mahmoud Al-Ayyoub, Yanyan Zhao, Bing Qin, Orphée De Clercq, Véronique Hoste, Marianna Apidianaki, Xavier Tannier, Natalia Loukachevitch, Evgeniy Kotelnikov, Nuria Bel, Salud María Jiménez-Zafra, and Gülşen Eryiğit. 2016. SemEval-2016 task 5: Aspect based sentiment analysis. In Proceedings of the 10th International Workshop on Semantic Evaluation (SemEval2016), pages 19-30, San Diego, California. Association for Computational Linguistics.

Maria Pontiki, Dimitris Galanis, Haris Papageorgiou, Suresh Manandhar, and Ion Androutsopoulos. 2015. SemEval-2015 task 12: Aspect based sentiment analysis. In Proceedings of the 9th International Workshop on Semantic Evaluation (SemEval 2015), pages 486-495, Denver, Colorado. Association for Computational Linguistics.

Maria Pontiki, Dimitris Galanis, John Pavlopoulos, Harris Papageorgiou, Ion Androutsopoulos, and Suresh Manandhar. 2014. SemEval-2014 task 4: Aspect based sentiment analysis. In Proceedings of the 8th International Workshop on Semantic Evaluation (SemEval 2014), pages 27-35, Dublin, Ireland. Association for Computational Linguistics. 
Peng Qi, Yuhao Zhang, Yuhui Zhang, Jason Bolton, and Christopher D. Manning. 2020. Stanza: A Python natural language processing toolkit for many human languages. In Proceedings of the 58th Annual Meeting of the Association for Computational Linguistics: System Demonstrations.

Alexander Rietzler, Sebastian Stabinger, Paul Opitz, and Stefan Engl. 2019. Adapt or get left behind: Domain adaptation through bert language model finetuning for aspect-target sentiment classification. arXiv preprint arXiv:1908.11860.

Youwei Song, Jiahai Wang, Tao Jiang, Zhiyue Liu, and Yanghui Rao. 2019. Attentional encoder network for targeted sentiment classification. arXiv preprint arXiv:1902.09314.

Nitish Srivastava, Geoffrey Hinton, Alex Krizhevsky, Ilya Sutskever, and Ruslan Salakhutdinov. 2014. Dropout: a simple way to prevent neural networks from overfitting. The Journal of Machine Learning Research, 15(1):1929-1958.

Kai Sun, Richong Zhang, Samuel Mensah, Yongyi Mao, and Xudong Liu. 2019. Aspect-level sentiment analysis via convolution over dependency tree. In Proceedings of the 2019 Conference on Empirical Methods in Natural Language Processing and the 9th International Joint Conference on Natural Language Processing (EMNLP-IJCNLP), pages 56835692.

Duyu Tang, Bing Qin, Xiaocheng Feng, and Ting Liu 2016. Effective LSTMs for target-dependent sentiment classification. In Proceedings of COLING 2016, the 26th International Conference on Computational Linguistics: Technical Papers, pages 3298 3307, Osaka, Japan. The COLING 2016 Organizing Committee.

Hao Tang, Donghong Ji, Chenliang Li, and Qiji Zhou. 2020. Dependency graph enhanced dualtransformer structure for aspect-based sentiment classification. In Proceedings of the 58th Annual Meeting of the Association for Computational Linguistics, pages 6578-6588, Online. Association for Computational Linguistics.

Ming Tu, Guangtao Wang, Jing Huang, Yun Tang, Xiaodong He, and Bowen Zhou. 2019. Multi-hop reading comprehension across multiple documents by reasoning over heterogeneous graphs. 57th Annual Meeting of the Association for Computational Linguistics, page pages 2704-2713.

Bailin Wang and Wei Lu. 2018. Learning latent opinions for aspect-level sentiment classification. In Thirty-Second AAAI Conference on Artificial Intelligence.

Kai Wang, Weizhou Shen, Yunyi Yang, Xiaojun Quan, and Rui Wang. 2020. Relational graph attention network for aspect-based sentiment analysis. In Proceedings of the 58th Annual Meeting of the Asso- ciation for Computational Linguistics, pages 3229 3238, Online. Association for Computational Linguistics.

Shuai Wang, Sahisnu Mazumder, Bing Liu, Mianwei Zhou, and Yi Chang. 2018. Target-sensitive memory networks for aspect sentiment classification. In Proceedings of the 56th Annual Meeting of the Association for Computational Linguistics (Volume 1: Long Papers), pages 957-967.

$\mathrm{Hu}$ Xu, Bing Liu, Lei Shu, and Philip S. Yu. 2019. Bert post-training for review reading comprehension and aspect-based sentiment analysis. In Proceedings of the 2019 Conference of the North American Chapter of the Association for Computational Linguistics.

Keyulu Xu, Chengtao Li, Yonglong Tian, Tomohiro Sonobe, Ken-ichi Kawarabayashi, and Stefanie Jegelka. 2018. Representation learning on graphs with jumping knowledge networks. In Proceedings of the 35th International Conference on Machine Learning, volume 80 of Proceedings of Machine Learning Research, pages 5453-5462. PMLR.

Chen Zhang, Qiuchi Li, and Dawei Song. 2019 Aspect-based sentiment classification with aspectspecific graph convolutional networks. In Proceedings of the 2019 Conference on Empirical Methods in Natural Language Processing and the 9th International Joint Conference on Natural Language Processing (EMNLP-IJCNLP), pages 4560-4570.

Yuhao Zhang, Peng Qi, and Christopher D Manning. 2018. Graph convolution over pruned dependency trees improves relation extraction. In Proceedings of the 2018 Conference on Empirical Methods in Natural Language Processing, pages 2205-2215.

Shiliang Zheng and Rui Xia. 2018. Left-center-right separated neural network for aspect-based sentiment analysis with rotatory attention. arXiv preprint arXiv:1802.00892. 